\title{
Direct observation of condensate and vortex confinement in nanostructured superconductors
}

\author{
M. Timmermans, L. Serrier-Garcia, M. Perini, ${ }^{*}$ J. Van de Vondel, and V. V. Moshchalkov \\ INPAC - Institute for Nanoscale Physics and Chemistry, Department of Physics and Astronomy, KU Leuven, \\ Celestijnenlaan 200D, B-3001 Leuven, Belgium \\ (Received 8 September 2015; revised manuscript received 18 January 2016; published 12 February 2016)
}

\begin{abstract}
In this work we report a scanning tunneling microscopy investigation of lithographically defined superconducting nanosquares. The obtained spectroscopic maps reveal the spatial evolution of both the superconducting condensate and the screening currents as a function of the applied magnetic field. The symmetry of the nanostructure is imposed on the condensate and it controls the distribution of the vortices inside the nanosquare. Our local study allows exploring the impact of small structural defects, omnipresent in these kind of structures, on both the supercurrent and vortex distribution. As a result, direct experimental evidence of vortex pinning and current crowding at the nanoscale has been obtained.
\end{abstract}

DOI: 10.1103/PhysRevB.93.054514

Confinement effects play an important role in different physical phenomena especially in quantum systems like Bose-Einstein condensates, superconductors, and superfluids. For example, the ability to structure superconducting devices at length scales comparable to the characteristic sizes (penetration dept $\lambda$ and coherence length $\xi$ ) of the condensate revealed a vast world of possibilities to explore quantum phenomena (e.g., creation of artificial atoms [1], induction of quantum phase slip lines [2], confinement effects [3], to name a few). In the latter example, theoretical modeling of these systems solving the Ginzburg-Landau (G-L) [4,5] or Bogoliubov-deGennes (B-dG) [6] equations for single and/or multiband mesoscopic superconductors has been done. All these simulations unveil the importance of confinement in mesoscopic superconducting systems. For example, the symmetry of the nanostructure will compete with the vortex-vortex interaction resulting in different vortex configurations compared to the triangular Abrikosov lattice, found in bulk superconductors [3-6].

These intriguing effects were experimentally investigated, using low temperature transport measurements, by probing the influence of nanostructuring on the superconductor/normal phase boundary [3]. Although these measurements proved the importance of size and shape they do not give sufficient local information about the spatial distribution of the superconducting condensate. Moreover, a different approach is needed to explore the condensate in the nondissipative (zero voltage) state. In order to tackle these issues a second set of experiments probes the magnetic field profiles, generated by the superconducting currents, by using magnetic field sensitive probes (e.g., Hall probe [7,8], scanning Hall probe microscopy [9], Bitter decoration [10], and scanning SQUID microscopy [11]). These techniques indeed visualized the symmetry-induced vortex configurations in superconducting nanostructures within the low confinement regime (i.e., nanostructure size $\sim \lambda \gg \xi$ ). The observed configurations are the results of the imposed boundary conditions and the repulsive magnetic interactions between vortices. In the strong confinement regime (i.e., nanostructure size $\sim \xi \ll \lambda$ ), the distribution of the superconducting condensate is governed by the kinetic energy of

\footnotetext{
*Current address: Department of Physics, University of Hamburg, D-20355 Hamburg, Germany.
}

the supercurrent distribution and magnetic interactions can be neglected [12]. Since most superconducting nanodevices work in this regime, it is of utmost importance to explore the strong confinement region with the appropriate, high resolution, tools.

This can be tackled using scanning tunneling microscopy (STM), as shown recently on very small in situ grown superconducting $\mathrm{Pb}$ islands [12-15]. These experiments indicate the high spatial resolution of STM to visualize both the superconducting condensate and the supercurrents. Nevertheless, these experiments share one common restriction, the sample surface needs to be very clean. As a result, alternative approaches are introduced to perform STM on superconducting nanodevices (e.g., Ref. [16]). In this work we tackled this challenge and present an extensive STM study on a set of gold covered $\mathrm{Mo}_{79} \mathrm{Ge}_{21}$ nanostructured squares in the strong confinement limit. We clearly show the importance of confinement effects and investigate the interplay between the induced screening currents, reflecting the $C 4$ symmetry, and the present vortex matter. Moreover, we have explored the importance of structural defects by combining topographic information with local mapping of the superconducting condensate. In addition, the presence of a surface barrier and vortex pinning gives us the possibility to explore the transition between different vortex states.

In order to fabricate $\mathrm{Mo}_{79} \mathrm{Ge}_{21}$ nanostructures with a high quality surface, allowing exploration with STM, we introduced the following sample preparation procedure. First, we evaporated a $33 \mathrm{~nm}$ Ge film on a conventional electron beam lithography pattern. The resulting nanostructure, consisting of a $5 \times 5 \mathrm{~mm}$ periodic array of square dots (width $=340 \mathrm{~nm}$ and period $=1.5 \mu \mathrm{m}$ ), will be used as a clean nanotemplate. After liftoff, we evaporated a $17 \mathrm{~nm}$ thick MoGe film using pulsed laser deposition. Finally, we directly covered the film with a thin $(5 \mathrm{~nm})$ gold layer to protect the $\mathrm{Mo}_{79} \mathrm{Ge}_{21}$ surface against oxidation. A schematic layout of the sample and an atomic force microscopy picture of the square Ge dots are shown in Fig. 1(a). The resulting structure combines the continuity of a plane film, needed to approach with the STM tip, with the introduction of the square boundary conditions explored in this work. As a proof of concept we show a low temperature STM image of a single island in Fig. 1(c). Since even a small amount of oxidation would be devastating for STM, the ability to visualize the island proves that, although granular, the $5 \mathrm{~nm} \mathrm{Au}$ top layer caps the $\mathrm{Mo}_{79} \mathrm{Ge}_{21}$ nanostructure. A detailed analysis 

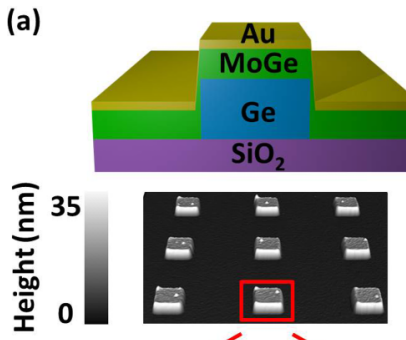

(c)

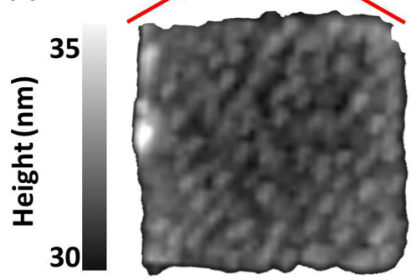

FIG. 1. Sample characterization. The upper panel of (a) shows a cross section of the structure of the islands and film. The lower panel pictures a topographic image of the periodic structure, using atomic force microscopy, whereas (c) zooms in on a single island, visualized by scanning tunneling microscopy. (b) Normalized conductance as a function of tip-sample voltage and temperature. The curves at higher temperature are shifted by a factor of 0.5 for clarity. (d) Zero bias conductance map of the complete island at $T=0.5 \mathrm{~K}$ and zero applied magnetic field.

on top and next to the islands indicates that the presence or absence of a Ge layer does not alter the growing conditions of the $\mathrm{Mo}_{79} \mathrm{Ge}_{21} / \mathrm{Au}$ bilayer. As a result, sample fabrication is only limited by the design of the lithographic mask and, therefore, various different superconducting structures and devices can be explored via this approach. The most important features influencing the choice of this material are: (i) The very weak intrinsic pinning in amorphous $\mathrm{Mo}_{79} \mathrm{Ge}_{21}$, confirmed by the material's extremely low critical currents. As such, the vortex-vortex and vortex-boundary interactions will play a dominant role [17]. (ii) The high value of $\lambda(\sim 500 \mathrm{~nm})$, compared to the dimensions of the nanoisland, ensuring demagnetization effects will not play a role [18]. (iii) The small value of $\xi(\sim 6 \mathrm{~nm})$ makes it possible to visualize spatial modulations in the superconducting condensate using STM [18]. All aforementioned ingredients allow a direct comparison with the theoretical models used to describe these systems $[4,19]$.

In a next step we investigated the superconducting condensate on top of the superconducting $\mathrm{Mo}_{79} \mathrm{Ge}_{21} / \mathrm{Au}$ islands using STM. The presented experimental data were all obtained on the normal metal $(\mathrm{Au})$ interface using a $\mathrm{Au}$ tunneling tip. In order to cope with the surface roughness all succeeding STM results are taken in current-imaging-tunneling spectroscopy (CITS) mode where an $I(V)$ curve is recorded at each pixel. The resolution of these maps roughly corresponds to $25 \mathrm{~nm}^{2}$ per pixel. Figure 1(c) shows the temperature dependence of the conductance, obtained by numerical differentiation of $I$ with respect to $V$, as a function of applied voltage. These curves show that the critical temperature is around $4.4 \mathrm{~K}$. This directly proves the presence of a proximity-induced local quasiparticle density of states in the Au layer, with a reduction of $T_{c}$ compared to a bare $\mathrm{Mo}_{79} \mathrm{Ge}_{21}$ thin film $\left(T_{c}=5.8 \mathrm{~K}\right)$.
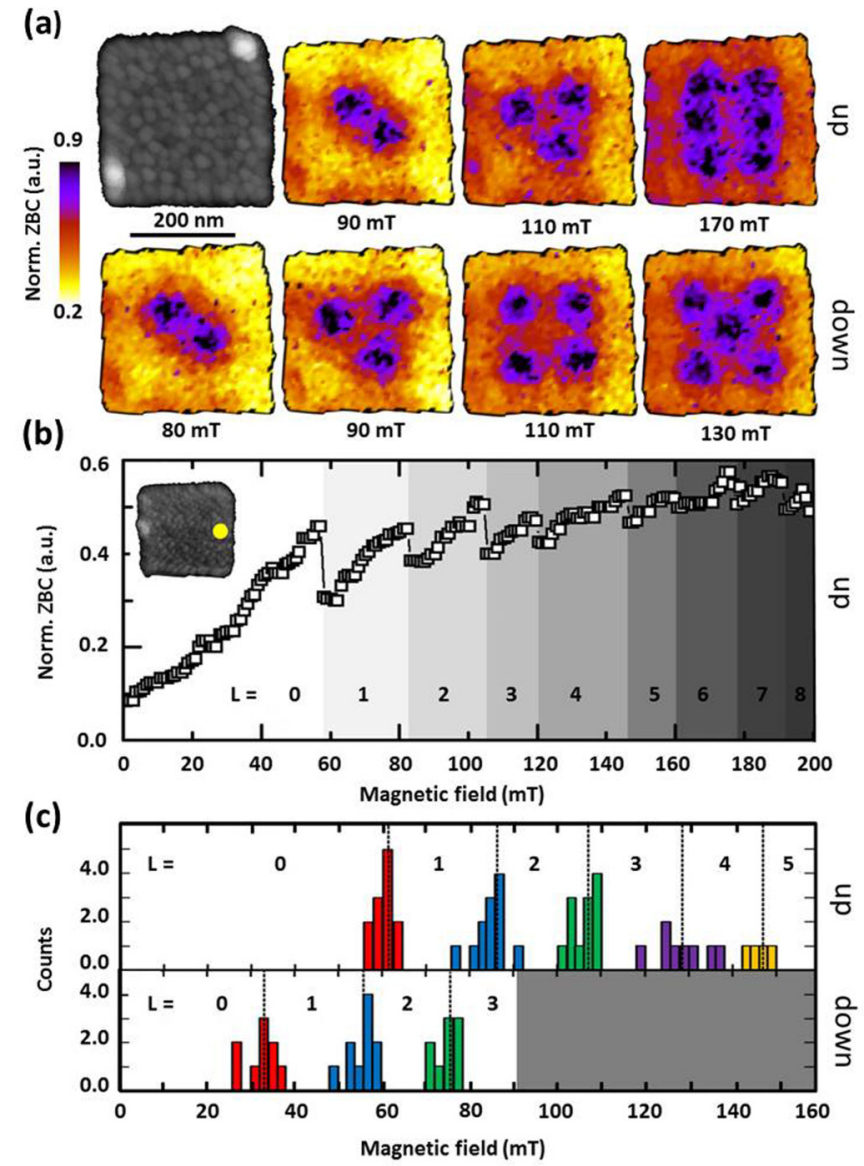

FIG. 2. Mapping of vortex distribution. All measurements were performed at $T=0.5 \mathrm{~K}$. (a) ZBC maps of a representative island for increasing (first row) and decreasing (second row) magnetic fields. (b) The magnetic field dependence of the zero bias conductance at a fixed position (marked by a yellow dot in inset). (c) The number of investigated squares that have a jump in vorticity at this magnetic field interval (interval size $=2 \mathrm{mT}$ ), while sweeping the field up (upper panel) or down (lower panel). Different colors relate the same steps in vorticity $L$.

Therefore, the $5 \mathrm{~nm} \mathrm{Au}$ layer protects the sample against oxidation, but also allows us to explore the superconducting gap underneath the Au layer [20]. From these data we also obtained the zero bias conductance (ZBC) giving a direct measure for the strength of the superconducting condensate at the tip position [12-14]. Figure 1(d) presents the distribution of the ZBC across the nanostructure at a temperature of $0.5 \mathrm{~K}$ and zero applied magnetic field. The map unveils only weak perturbations in the $\mathrm{ZBC}$ unrelated to the topographic image [see Fig. 1(c)]. This shows that, although the $\mathrm{Mo}_{79} \mathrm{Ge}_{21} / \mathrm{Au}$ bilayer is clearly irregular, an almost uniform condensate is present across the entire nanoisland.

Figure 2 gives a direct observation of the effects of the boundary conditions (and their symmetry) on the vortex distribution inside the islands. As shown in Fig. 2(a), the vortex configurations for both increasing (top row) and decreasing (bottom row) magnetic fields are clearly ruled by the $C 4$ symmetry of the nanoislands. The obtained vortex positions are in a very good agreement with theoretically predicted distributions [19,21]. This is a direct confirmation of the 
predicted, symmetry induced vortex distribution in superconducting nanostructures. No indication of a symmetry induced antivortex, predicted by Chibotaru and co-workers [4], was found. This absence can be explained by the low measurement temperature needed to resolve vortices and the inherently present sample imperfections [22]. Moreover, the use of a periodic array of islands allows us to explore the dependence of the superconducting properties on subtle differences among islands unavoidable in lithographically defined nanostructures.

In order to compare a set of islands, in a reasonable time frame, one can measure the transitions between vortex patterns by placing the tip at the border as indicated by the yellow dot in the inset of Fig. 2(b). The transitions between different vortex states are visualized by a change in the ZBC conductance while sweeping the magnetic field up [12,13,23]. In order to reduce the noise level, an average over 16 CITS curves is taken, generating the curve shown in Fig. 2(b). The number of vortices inside the islands is given by the vorticity $L$. Until 58 $\mathrm{mT}$ a clear increase of the normalized ZBC is observed related to the induced screening currents, well know as being able to reduce the superconducting gap at the sample border. At $58 \mathrm{mT}$ an abrupt reduction of the normalized $\mathrm{ZBC}$ is observed, which is attributed to the entrance of a vortex inside the nanoisland, accompanied by a reduction of the supercurrents running at the sample edge [12]. Smaller jumps, all related to different changes in vorticity $(L \rightarrow L+1)$, can be found at 82,104 , 119, 146, 160, 178, and $192 \mathrm{mT}$. In Fig. 2(c) the exact magnetic field at which jumps in vorticity occur are summarized for different islands, once while increasing the magnetic field (bottom panel) and once while decreasing it (upper panel). The small spread in distribution for each $L \rightarrow L+1$ transition indicates that in the first approximation the different squares have very similar superconducting properties, although minor variations in the shape of the islands cannot be neglected [24]. In the case of a square island with a lateral size of $340 \mathrm{~nm}$ a magnetic field of $\pm 18 \mathrm{mT}$ is needed to increase the induced flux quanta by one. However, in mesoscopic structures the values needed to increase vorticity are always bigger due to the strong effect of confinement, e.g., [5]. At high vorticities, the confinement effect becomes less influential and indeed the experimentally observed width of the stability region is $\pm 20 \mathrm{mT}(L=3)$, which is in very good agreement with the expected field value. Comparing the exact magnetic field of the entrance and exit of one vortex reveals a strong hysteresis [bottom versus top panel in Fig. 2(c)]. This proves the presence of a so-called surface barrier and indicates the importance of the edge quality $[13,21]$.

In the final part we will use the power of the technique to explore how imperfections inside and at the edge of the islands alter the exact vortex distribution and probe the evolution between different vortex distributions as a function of the magnetic field. Figure 3(a) (top row) shows a topographic image of an island revealing an imperfection (hillock) close to the center of the island. It is important to note that a ZBC map of the island shows no depleted region at this exact location. This feature should act as a pinning center for vortices [25], as clearly seen by visualizing the vortex distribution in this island, given by the circle $(L=1)$, line $(L=2)$, and triangles ( $L=3$ ). Although the first vortex is positioned in the middle of the island, one vortex is always trapped at the defect for

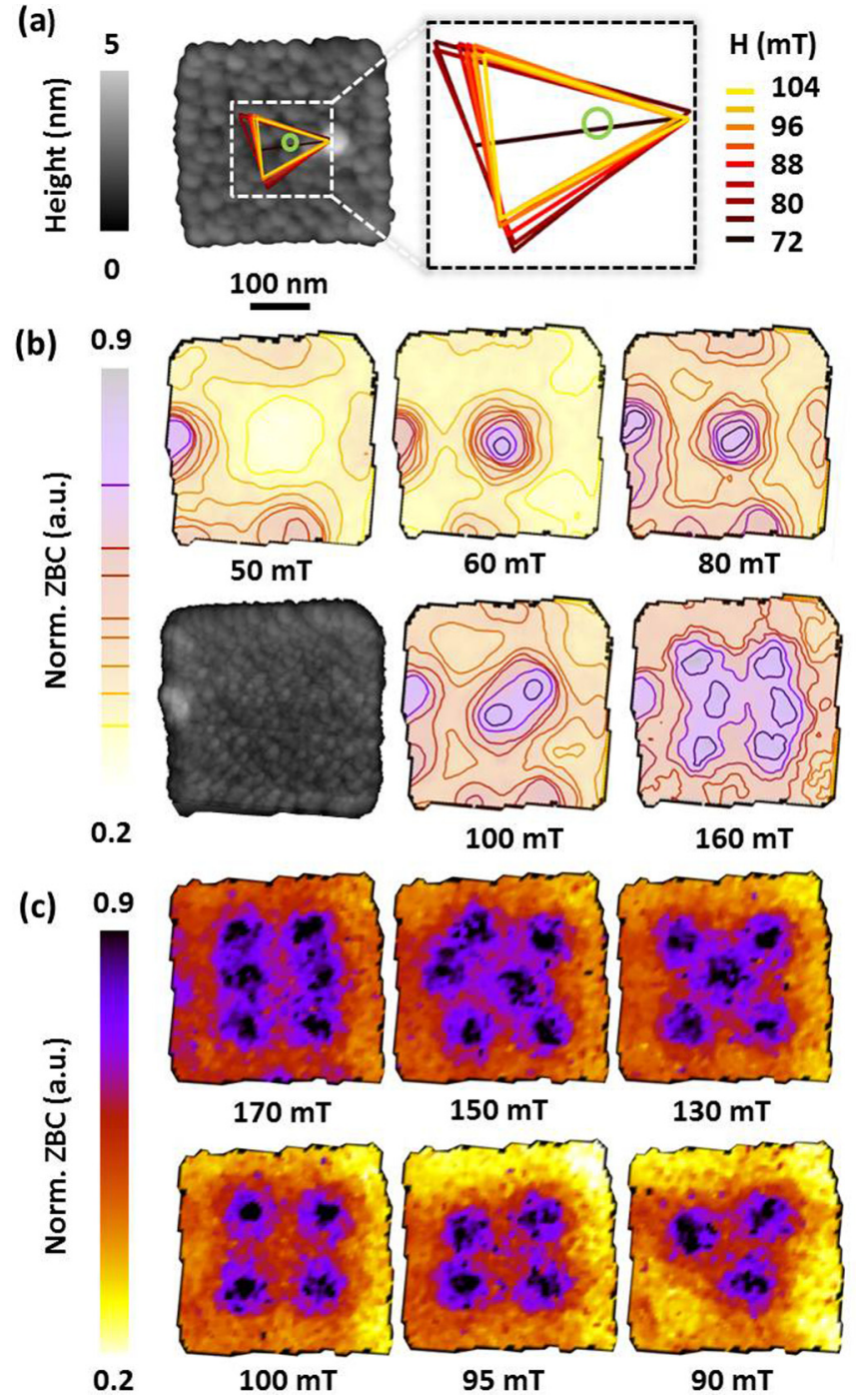

FIG. 3. (a) In grayscale the topography of the square with close to the center a spurious defect. The circle, line, and triangles represent, respectively, 1, 2, and 3 vortice(s). The color scale defines the applied magnetic field. (b) In grayscale the topography of the square with close to the edge a spurious defect. The contour plots mark the normalized ZBC values. (c) ZBC maps at different magnetic field values of the island shown topographically in Fig. 2(a).

higher vorticities. This indicates that pinning is weak, but has a clear impact on the distribution of the vortices. For example, in the $L=2$ case one vortex is trapped at the defect position and the vortex pair is aligned horizontally instead of diagonally. The latter is expected theoretically [21] and was confirmed in "clean" islands [Fig. 2(a)]. Another example can be observed upon decreasing the magnetic field for $L=3$. The vortex triplet rotates and expands, shown by the triangular representation, around a fixed axis located at the hillock.

Next we investigate the impact on the condensate of a similar corrugation located at the edge of the island [Fig. 3(b)]. When increasing the magnetic field until $50 \mathrm{mT}(L=0)$ screening currents are induced at the sample edge. This results in an increase in the $\mathrm{ZBC}$ at the edge of the sample. Indeed, the pattern indicated by the contour plot follows the $C 4$ symmetry, which confirms that the boundary conditions are imposed on 
the superconducting condensate. In addition, at the corrugation a much stronger depletion is visible. We believe that this enhanced reduction can only be explained by an increased density of the screening currents due to the small hillock at the sample border. Indeed, previous theoretical calculations, regarding the impact of defects on the depletion are in very good agreement with our observations [26]. As a result, this is the first direct observation of the current crowding effect at the nanoscale [27,28]. In order to strengthen our claim we also present the ZBC maps at $60 \mathrm{mT}$. At this field value a vortex enters leading to an overall reduction of the screening currents at the edge, therefore the $\mathrm{ZBC}$ should be decreased at the sample edge. This is indeed confirmed, nevertheless the increased effect due to the corrugation is still present. At higher magnetic fields (e.g., $160 \mathrm{mT}$ ), the defect distorts the vortex distributions, again in very good agreement with theoretical predictions [26].

Finally we can use our approach to explore the exit and/or entrance of a single vortex, which is highly relevant in order to probe the energy barrier existing between different vorticities [29]. Two examples are given in Fig. 3(c) for the $L=4$ to $L=3$ (bottom panel) and $L=6$ to $L=5$ (top panel) transition. In both cases the initial state has a symmetrical distribution. By lowering the magnetic field one vortex wants to leave the square, but this exit is prohibited by the surface barrier. Still a transition sets in and the vortex configuration gently moves to a distorted vortex pattern mimicking the $L=3$ and $L=5$ distribution. At lower fields this vortex has left the sample, and a stable configuration is settled.

To conclude, we performed STM studies on lithographically patterned nanosquares of $\mathrm{Mo}_{79} \mathrm{Ge}_{21} / \mathrm{Au}$ bilayers. We investigated the vortex distribution at different magnetic field values and unambiguously demonstrated the importance of the effects of the sample symmetry on the obtained vortex patterns. Moreover, minor variations exist between different islands which we believe are related to sample imperfections unavoidable in this type of sample fabrication. These imperfections allow us to explore current crowding, vortex pinning effects, and evolution of the vortex distribution in these nanoislands. As such, the presented approach can now be used to explore various superconducting phenomena predicted for superconducting nanostructures (e.g., the presence of symmetry induced vortex-antivortex pairs).

We thank T. Samuely for his help with the start-up experiments. This work was supported by the Methusalem Funding 08/05 by the Flemish Government, the Fund for Scientific Research-Flanders (FWO-Vlaanderen), and the MP1201 COST-action.
[1] J. Q. You and F. Nori, Nat. Phys. 474, 589 (2011).

[2] A. Bezryadin, C. N. Lau, and M. Tinkham, Nature (London) 404, 971 (2000).

[3] V. V. Moshchalkov, L. Gielen, C. Strunk, R. Jonckheere, X. Qiu, C. Van Haesendonck, and Y. Bruynseraede, Nature (London) 373, 319 (1995).

[4] L. F. Chibotaru, A. Ceulemans, V. Bruyndoncx, and V. V. Moshchalkov, Nature (London) 408, 833 (2000).

[5] L. F. Chibotaru, A. Ceulemans, M. Morelle, G. Teniers, C. Carballeira, and V. V. Moshchalkov, J. Math. Phys. 46, 095108 (2005).

[6] L.-F. Zhang, L. Covaci, M. V. Milosěvić, G. R. Berdiyorov, and F. M. Peeters, Phys. Rev. Lett. 109, 107001 (2012).

[7] A. K. Geim, I. V. Grigorieva, S. V. Dubonos, J. G. S. Lok, J. C. Maan, A. E. Filippov, and F. M. Peeters, Nature (London) 390, 259 (1997).

[8] M. Morelle, J. Bekaert, and V. V. Moshchalkov, Phys. Rev. B 70, 094503 (2004).

[9] T. Nishio, Q. Chen, W. Gillijns, K. De Keyser, K. Vervaeke, and V. V. Moshchalkov, Phys. Rev. B 77, 012502 (2008).

[10] H. J. Zhao, V. R. Misko, F. M. Peeters, V. Oboznov, S. V. Dubonos, and I. V. Grigorieva, Phys. Rev. B 78, 104517 (2008).

[11] N. Kokubo, S. Okayasu, A. Kanda, and B. Shinozaki, Phys. Rev. B 82, 014501 (2010).

[12] T. Cren, D. Fokin, F. Debontridder, V. Dubost, and D. Roditchev, Phys. Rev. Lett. 102, 127005 (2009).

[13] T. Nishio, T. An, A. Nomura, K. Miyachi, T. Eguchi, H. Sakata, S. Lin, N. Hayashi, N. Nakai, M. Machida, and Y. Hasegawa, Phys. Rev. Lett. 101, 167001 (2008).

[14] T. Cren, L. Serrier-Garcia, F. Debontridder, and D. Roditchev, Phys. Rev. Lett. 107, 097202 (2011).

[15] D. Roditchev, C. Brun, L. Serrier-Garcia, J. C. Cuevas, V. H. L. Bessa, M. V. Milosěvić, F. Debontridder, V. Stolyarov, and T. Cren, Nat. Phys. 11, 332 (2015).
[16] J. Senzier, P. S. Luo, and H. Courtois, Appl. Phys. Lett. 90, 043114 (2007).

[17] B. L. T. Plourde, D. J. Van Harlingen, D. Yu. Vodolazov, R. Besseling, M. B. S. Hesselberth, and P. H. Kes, Phys. Rev. B 64, 014503 (2001).

[18] M. Motta, F. Colauto, J. I. Vestgården, J. Fritzsche, M. Timmermans, J. Cuppens, C. Attanasio, C. Cirillo, V. V. Moshchalkov, J. Van de Vondel, T. H. Johansen, W. A. Ortiz, and A. V. Silhanek, Phys. Rev. B 89, 134508 (2014).

[19] B. J. Baelus, A. Kanda, N. Shimizu, K. Tadano, Y. Ootuka, K. Kadowaki, and F. M. Peeters, Phys. Rev. B 73, 024514 (2006).

[20] A. K. Gupta, L. Crétinon, N. Moussy, B. Pannetier, and H. Courtois, Phys. Rev. B 69, 104514 (2004).

[21] B. J. Baelus and F. M. Peeters, Phys. Rev. B 65, 104515 (2002).

[22] L.-F. Zhang, L. Covaci, M. V. Milosěvić, G. R Berdiyorov, and F. M. Peeters, Phys. Rev. B 88, 144501 (2013).

[23] A. Kanda, B. J. Baelus, F. M. Peeters, K. Kadowaki, and Y. Ootuka, Phys. Rev. Lett. 93, 257002 (2004).

[24] Y. Kuroda, S. Hatsumi, Y. Ootuka, and A. Kanda, Physica C 470, 1145 (2010).

[25] M. Van Bael, Ph.D. thesis, KU Leuven, 1998.

[26] B. J. Baelus, K. Kadowaki, and F. M. Peeters, Phys. Rev. B 71, 024514 (2005).

[27] O.-A. Adami, D. Cerbu, D. Cabosart, M. Motta, J. Cuppens, W. A. Ortiz, V. V. Moshchalkov, B. Hackens, R. Delamare, J. Van de Vondel, and A. V. Silhanek, App. Phys. Lett. 102, 052603 (2013).

[28] H. L. Hortensius, E. F. C. Driessen, T. M. Klapwijk, K. K. Berggren, and J. R. Clem, Appl. Phys. Lett. 100, 182602 (2012).

[29] B. J. Baelus, F. M. Peeters, and V. A. Schweigert, Phys. Rev. B 63, 144517 (2001) 